\title{
SOME THEOREMS ON RECTILINEAR CONGRUENCES AND TRANSFORMATIONS OF SURFACES
}

\author{
BY \\ CHENKUO PA
}

1. Let $S$ be a non-developable analytic surface in ordinary space and $L$ a rectilinear congruence with each of its generators passing through a corresponding point of $S$ but not tangent to $S$. For any given surface these congruences depend on two arbitrary functions. In this note, we obtain some properties on the relations between a surface and its associate congruences above defined and deduce some new transformations of certain kinds of surfaces, which seems to be quite different from the classical transformations of these surfaces. The main results may be formulated as follows:

THEOREM I. Let $S$ be a surface and $L$ a rectilinear $W$-congruence with each of its generators passing through the corresponding point of $S$ but not tangent to $S$. If the asymptotic net of $S$ correspond to that of the focal sheets of $L$, then $S$ is an $R$-surface and $L$ is an $R$-congruence with its developables cutting $S$ in an $R$-net. Conversely, for a given $R$-surface, there exist $\infty^{4} R$-congruences having the above properties.

THEOREM II. Let $S$ be a surface and $L$ a rectilinear congruence conjugate to $S$, and let $S^{\prime}$ be a surface generated by the harmonic conjugate point of a point of $S$ with respect to the foci of $L$ on the corresponding generator through the point of $S$. If the asymptotic nets of $S$ and $S^{\prime}$ are in correspondence, then both $S$ and $S^{\prime}$ are Jonas surfaces and the developables of $L$ cut both $S$ and $S^{\prime}$ in Jonas nets. Conversely, for a given Jonas surface $S$, there exist $\infty^{8}$ rectilinear congruences conjugate to $S$ which satisfy the above conditions.

2. Let $u, v$ be the asymptotic parameters of $S$ and $x^{i}(i=1,2,3,4)$ the projective homogeneous coordinates of a generic point of $S$. The fundamental differential equations of $S$ are

$$
\begin{aligned}
& x_{u u}=\beta x_{v}+\theta_{u} x_{u}+p_{11} x, \\
& x_{v v}=\gamma x_{u}+\theta_{v} x_{v}+p_{22} x,
\end{aligned}
$$

with the following conditions of integrability:

$$
\begin{aligned}
& \left(\theta_{u v}+\beta \gamma\right)_{v}+\gamma \pi_{11}=\pi_{22 u}+p_{22 u}+\gamma p_{11}+\theta_{v}\left(\theta_{u v}+\beta \gamma\right), \\
& \left(\theta_{u v}+\beta \gamma\right)_{u}+\beta \pi_{22}=\pi_{11 v}+p_{11 v}+\beta p_{22}+\theta_{u}\left(\theta_{u v}+\beta \gamma\right), \\
& \pi_{11 v v}-\beta \pi_{22 v}-2 \beta_{v} \pi_{22}+\theta_{u} \pi_{22 u}=\pi_{22 u u}-\gamma \pi_{11 u}-2 \gamma_{u} \pi_{11}+\theta_{v} \pi_{11 v},
\end{aligned}
$$

Presented to the Society, September 10, 1948; received by the editors February 4, 1948. 
where we have put

$$
\pi_{11}=\beta_{v}+\beta \theta_{v}+p_{11}, \quad \pi_{22}=\gamma_{u}+\gamma \theta_{u}+p_{22} .
$$

The congruence $L$ above defined can be expressed by

$$
\bar{x}=x+R\left(x_{u v}-l_{1} x_{v}-l_{2} x_{u}\right),
$$

whose developables cut $S$ in curves whose differenential equation is

$$
\begin{gathered}
\left(\pi_{11}-l_{1 u}+l_{1} \theta_{u}-l_{2} \beta-l_{1}^{2}\right) d u^{2}+\left(-\pi_{22}+l_{2 v}-l_{2} \theta_{v}+l_{1} \gamma+l_{2}^{2}\right) d v^{2} \\
+\left(l_{2 u}-l_{1 v}\right) d u d v=0 .
\end{gathered}
$$

The values of $R$ corresponding to the foci are found to be:

$$
\frac{1}{R^{2}}+\frac{1}{R}\left[2\left(\beta \gamma+\theta_{u v}\right)-\left(l_{2 u}+l_{1 v}+2 l_{1} l_{2}\right)\right]+\cdots=0 .
$$

With the aid of (5) we find that, if $L$ is conjugate to $S$, then

$$
l_{2 u}=l_{1 v} \text {. }
$$

3. Proof of the Theorem I. To prove Theorem I we require the following two lemmas.

LEMMA 1. A necessary and sufficient condition that a congruence $L$ should be conjugate to a surface $S$ is that the asymptotic u-tangents (v-tangents) of $S$ will intersect the corresponding v-tangents (u-tangents) of the surface $S^{\prime}$, where $S^{\prime}$ denotes the surface generated by the harmonic conjugate point of the point of $S$ with respect to the foci of $L$ on the corresponding generator.

It is clear from (6) that the surface $S^{\prime}$ is defined by

$$
x^{\prime}=H x+x_{u v}-l_{1} x_{v}-l_{2} x_{u},
$$

where we have put

$$
H=-\left(\theta_{u v}+\beta \gamma\right)+l_{1} l_{2}+\left(l_{2 u}+l_{1 v}\right) / 2 .
$$

By differentiating (1), we have.

$$
\begin{aligned}
& x_{u u v}=\left(\theta_{u v}+\beta \gamma\right) x_{u}+\pi_{11} x_{v}+\left(p_{11 v}+\beta p_{22}\right) x+\theta_{u} x_{u v}, \\
& x_{u v v}=\pi_{22} x_{u}+\left(\theta_{u v}+\beta \gamma\right) x_{v}+\left(p_{22 u}+\gamma p_{11}\right) x+\theta_{v} x_{u v},
\end{aligned}
$$

whence follows the expression for $x_{u}^{\prime}$ :

$$
x_{u}^{\prime}=\left(\theta_{u}-l_{1}\right) x^{\prime}+\left(\pi_{11}-l_{1 u}-l_{2} \beta+l_{1} \theta_{u}-l_{1}^{2}\right) x_{v}+2^{-1}\left(l_{1 v}-l_{2 u}\right) x_{u}+p x,
$$

the exact value of $p$ being inutile for our subsequent discussion.

If the $u$-tangents of $S^{\prime}$ intersect the corresponding $v$-tangents of $S$, then 


$$
\left(x^{\prime}, x_{u}^{\prime}, x, x_{v}\right)=0 .
$$

In view of the preceding relations, we see that the last equation is satisfied if and only if

$$
l_{1 v}=l_{2 u},
$$

which proves the lemma.

LEMMA $2\left(^{1}\right)$. If $x, x^{\prime}$ are conjugate with respect to the foci of a congruence $\left(x, x^{\prime}\right)$, and if $\left(x, x^{\prime}\right)$ is $W$, then the lines $\left(x, x_{u}\right)$ and $\left(x^{\prime}, x_{v}^{\prime}\right)$ meet, and also the lines $\left(x, x_{v}\right)$ and $\left(x^{\prime}, x_{u}^{\prime}\right)$ intersect, where $u$ and $v$ are the asymptotic parameters of the focal surfaces.

It is clear from these lemmas that if the surface $S$ and the congruence $L$ have the properties stated in Theorem $\mathrm{I}$, the congruence $L$ must be conjugate to $S$, and we obtain as a necessary consequence equation (7). That is, the differential form

$$
l_{1} d u+l_{2} d v
$$

is exact. By changing the factor of proportionality of the homogeneous coordinates, we can make

$$
l_{1}=l_{2}=0 \text {. }
$$

After this reduction the congruence $L$ is given by

$$
\bar{x}=x+R x_{u v},
$$

or better, by the line coordinates

$$
r=\left(x, x_{u v}\right) .
$$

By means of the equations (1), (2) and (10) we have

$$
\begin{aligned}
r_{u}= & \left(x_{u}, x_{u v}\right)+\left(\theta_{u v}+\beta \gamma\right)\left(x, x_{u}\right)+\pi_{11}\left(x, x_{v}\right)+\theta_{u}\left(x, x_{u v}\right), \\
r_{v}= & \left(x_{v}, x_{u v}\right)+\pi_{22}\left(x, x_{u}\right)+\left(\theta_{u v}+\beta \gamma\right)\left(x, x_{v}\right)+\theta_{v}\left(x, x_{u v}\right), \\
r_{u v}= & \theta_{v}\left(x_{u}, x_{u v}\right)+\theta_{u}\left(x_{v}, x_{u v}\right)+\left[\pi_{22 u}+\theta_{u} \pi_{22}+\theta_{v}\left(\theta_{u v}+\beta \gamma\right)\right]\left(x, x_{u}\right) \\
& +\left[\pi_{11 v}+\theta_{v} \pi_{11}+\theta_{u}\left(\theta_{u v}+\beta \gamma\right)\right]\left(x, x_{v}\right) \\
& +\left(\beta \gamma+2 \theta_{u v}+\theta_{u} \theta_{v}\right)\left(x, x_{u v}\right) .
\end{aligned}
$$

Eliminating $\left(x_{u}, x_{u v}\right),\left(x_{v}, x_{u v}\right),\left(x, x_{u v}\right)$ from (12) and (13) we obtain (14) $r_{u v}-\theta_{v} r_{u}-\theta_{u} r_{v}+\left(\theta_{u} \theta_{v}-2 \theta_{u v}-\beta \gamma\right) r-\pi_{22 u}\left(x, x_{u}\right)-\pi_{11 v}\left(x, x_{v}\right)=0$.

From a classical theorem of Darboux we know the conditions that $L$ should be a $W$-congruence are

(1) G. Fubini, On a property of W-congruences, Ann. of Math. vol. 41 (1940) p. 360. 


$$
\pi_{22 u}=\pi_{11 v}=0 .
$$

Since the form

$$
\pi=\pi_{11} d u^{2}+\pi_{22} d v^{2}
$$

is intrinsic, a change of asymptotic parameters:

$$
\bar{u}=\int\left(\pi_{11}\right)^{1 / 2} d u, \quad \bar{v}=\int\left(\pi_{22}\right)^{1 / 2} d v
$$

gives $\left({ }^{2}\right)$

$$
\pi_{11}=\pi_{22}=1 .
$$

Substituting (16) in the condition of integrability $\left(2_{3}\right)$ we obtain immediately that

$$
\beta_{v}=\gamma_{u},
$$

which is the characteristic equation of an $R$-surface.

It is thus clear, by (14), that the congruence $L$ is an $R$-congruence and, by (5), that the developables of $L$ cut $S$ in curves:

$$
d u^{2}-d v^{2}=0,
$$

which is the $R$-net. Thus we have established the first part of Theorem I.

As to the converse of Theorem I, we shall determine, for a given $R$-surface, all the congruences having the quoted properties.

In view of the preceding discussion we may conclude that for a given $R$-surface $\beta_{v}=\gamma_{u}$, if we could, by changing the factor of proportionality $x^{\prime}=\rho x$, reduce $\pi_{11}=\pi_{22}=k(k \neq 0$, arbitrary constant), then the congruence formed by the lines joining $x$ and $(\rho x)_{u v}$ is the required congruence. If $\beta^{\prime}, \gamma^{\prime}, \theta_{u}^{\prime}, \theta_{v}^{\prime}$, $p_{11}^{\prime}, p_{22}^{\prime}$ are the new fundamental quantities of $S$, we obtain by a simple calculation the following relations:

and

$$
\begin{aligned}
\beta^{\prime}=\beta, \quad \gamma^{\prime} & =\gamma, \quad \theta_{u}^{\prime}=\theta_{u}-2 \rho_{u} / \rho, \quad \theta_{v}^{\prime}=\theta_{v}-2 \rho_{v} / \rho, \\
p_{11}^{\prime} & =p_{11}+\beta \rho_{v} / \rho+\theta_{u} \rho_{u} / \rho-\rho_{u u} / \rho, \\
p_{22}^{\prime} & =p_{22}+\gamma \rho_{u} / \rho+\theta_{v} \rho_{v} / \rho-\rho_{v v} / \rho,
\end{aligned}
$$

$$
\begin{aligned}
& \pi_{11}^{\prime}=\pi_{11}+\theta_{u} \rho_{u} / \rho-\beta \rho_{v} / \rho-\rho_{u u} / \rho, \\
& \pi_{22}^{\prime}=\pi_{22}+\theta_{v} \rho_{v} / \rho-\gamma \rho_{u} / \rho-\rho_{v v} / \rho .
\end{aligned}
$$

Therefore the required function $\rho$ must be a solution of the following system of differential equations:

(2) $\pi_{11} \pi_{22} \neq 0$, for, if $\pi_{11}=\pi_{22}=0$, the developables of $L$ are indeterminate and the focal surfaces reduce to a fixed point, and if either $\pi_{11}=0$ or $\pi_{22}=0$ the developables reduce to $d v^{2}=0$, or $d u^{2}=0$, which is evidently not conjugate to $S$. 


$$
\begin{aligned}
& \rho_{u u}=-\beta \rho_{v}+\theta_{u} \rho_{u}+\left(\pi_{11}-k\right) \rho, \\
& \rho_{v v}=-\gamma \rho_{u}+\theta_{v} \rho_{v}+\left(\pi_{22}-k\right) \rho .
\end{aligned}
$$

Because of $(2)\left({ }^{3}\right)$ the last system is completely integrable. Hence for every constant $k$, there exist $\infty^{4}$ congruences having the required properties.

This completes the proof of Theorem I.

Similarly, we may consider the correlative theorem of Theorem I.

4. We shall give here some simple applications of Theorem I. It is known that the projective normal congruence of a surface $S$ is conjugate to $S$. If this congruence is $W$ and the asymptotic curves of its focal surfaces correspond to those of $S$, then $S$ belongs to a special class of $R$-surfaces with the following characteristic conditions:

$$
p_{11}+\beta_{v}+\beta \theta_{v}=1, \quad p_{22}+\gamma_{u}+\gamma \theta_{u}=1,
$$

where

$$
\theta=\log \beta \gamma .
$$

When the canonical line $C(\lambda)(\lambda \neq 0)$ of the first canonical pencil of a surface $S$ forms a $W$-congruence whose asymptotic curves of its focal surfaces correspond to those of $S$, the surface is isothermally asymptotic and belongs to a class of $R$-surfaces; its characteristic equations are

$$
\beta=\gamma, \quad \theta=2(3 \lambda+1) \log \beta, \quad \pi_{11 v}=\pi_{22 u}=0 .
$$

Surfaces of this class may be considered as generalizations of the surfaces whose canonical line $C(\lambda)$ passes through a fixed point $\left(^{4}\right)$.

When the congruence $L$ and the reciprocal congruence $L^{\prime}$ of $L$ with respect to $S$ both form $W$-congruences and the asymptotic curves of the focal sheets of each congruence correspond to those of $S, S$ belongs to a class of surfaces defined by

$$
\pi_{11}=\pi_{22}=1, \quad p_{11 v}=p_{22 u}=0 .
$$

We shall discuss these classes of surfaces on another occasion.

5. Proof of Theorem II. The surface $S^{\prime}$ stated in Theorem II is the locus of the point

$$
e^{\theta} x^{\prime}=-\left(\theta_{u v}+\beta \gamma\right) x+x_{u v} .
$$

By a simple calculation, we find that

(3) For, equation (2) is also valid under the substitution

$$
\left(\begin{array}{rrrr}
\beta & \gamma & p_{11} & p_{22} \\
-\beta & -\gamma & \pi_{11} & \pi_{22}
\end{array}\right)
$$

(4) For the latter class $\beta=\gamma, \theta=2(3 \lambda+1) \log \beta, \pi_{11}=\pi_{22}=0$, see Fubini and Cech, Geometria proiettiva differenziale, vol. 1, pp. 160-170. 


$$
\begin{aligned}
e^{\theta} x_{u}^{\prime}= & \left(\pi_{22} \beta-\pi_{11 v}\right) x+\pi_{11} x_{v}, \\
e^{\theta} x_{v}^{\prime}= & \left(\pi_{11} \gamma-\pi_{22 u}\right) x+\pi_{22} x_{u}, \\
e^{\theta} x_{u u}^{\prime}= & \left(\pi_{22 u} \beta+\pi_{22} \beta_{u}-\pi_{11 u v}-\pi_{22} \theta_{u} \beta+\theta_{u} \pi_{11 v}\right) x \\
& +\left(\pi_{22} \beta-\pi_{11 v}\right) x_{u}+\left(\pi_{11 u}-\theta_{u} \pi_{11}\right) x_{v}+\pi_{11} x_{u v} .
\end{aligned}
$$

If the asymptotic curves $v$ of $S$ correspond to those of $S^{\prime}$, then $x^{\prime}$ satisfies an equation of the form

$$
x_{u u}^{\prime}=\beta^{\prime} x_{v}^{\prime}+\theta_{u}^{\prime} x_{u}^{\prime}+p_{11}^{\prime} x^{\prime} \text {. }
$$

Substituting the values of $x_{u u}^{\prime}, x_{u}^{\prime}, x_{v}^{\prime}, x^{\prime}$ given by (22) and (23) in (24), we obtain

$$
\begin{aligned}
& \beta^{\prime}=\beta-\pi_{11 v} / \pi_{22}, \\
& \theta_{u}^{\prime}=-\theta_{u}+\pi_{11 u} / \pi_{11}, \\
& p_{11}^{\prime}=\pi_{11}, \\
& \beta^{\prime}\left(\pi_{11} \gamma-\pi_{22 u}\right)+\theta_{u}^{\prime}\left(\pi_{22} \beta-\pi_{11 v}\right)-p_{11}^{\prime}\left(\theta_{u v}+\beta \gamma\right) \\
& \quad=\pi_{22 u} \beta+\pi_{22} \beta_{u}-\pi_{11 u v}-\theta_{u} \pi_{22} \beta+\theta_{u} \pi_{11 v} .
\end{aligned}
$$

Eliminating $\beta^{\prime}, \theta_{u}^{\prime}, p_{11}^{\prime}$ from these equations and reducing, we get the required condition as follows:

$$
\begin{aligned}
\left(\frac{\pi_{22}}{\pi_{11}} \beta\right)_{u}-\frac{\partial^{2} \log \pi_{11}}{\partial u \partial v}= & -\theta_{u v}+\frac{\partial \log \pi_{11}}{\partial v} \frac{\partial \log \pi_{22}}{\partial u} \\
& -\beta \frac{\pi_{22 u}}{\pi_{11}}-\gamma \frac{\pi_{11 v}}{\pi_{22}}
\end{aligned}
$$

Similarly, a necessary and sufficient condition that the asymptotic curves $u$ correspond is

$$
\begin{aligned}
& \left(\frac{\pi_{11}}{\pi_{22}} \gamma\right)_{v}-\frac{\partial^{2} \log \pi_{22}}{\partial u \partial v}=-\theta_{u v}+\frac{\partial \log \pi_{11}}{\partial v} \frac{\partial \log \pi_{22}}{\partial u} \\
& -\beta \frac{\pi_{22 u}}{\pi_{11}}-\gamma \frac{\pi_{11 v}}{\pi_{22}} .
\end{aligned}
$$

From (26) and (27) we get immediately the following relation:

$$
\left(\frac{\pi_{22}}{\pi_{11}} \beta\right)_{u}-\left(\frac{\pi_{11}}{\pi_{22}} \gamma\right)_{v}-\frac{\partial^{2} \log \pi_{11} / \pi_{22}}{\partial u \partial v}=0,
$$

which shows that $\left(^{5}\right)$ the developables of $L$ cut $S$ in a conjugate net with equal (5) Cf. Fubini and Cech, loc. cit. p. 105. 
point invariants.

The second of $\left(25_{1}\right)$ implies that the equations (26) and (27) can be written in the form

$$
\theta_{u}^{\prime}=-\theta_{u}+\pi_{11 u} / \pi_{11}, \quad \theta_{v}^{\prime}=-\theta_{v}+\pi_{22 v} / \pi_{22} .
$$

Combining the last two relations we get as a condition of integrability of (26) and (27)

$$
\frac{\partial^{2} \log \pi_{11} / \pi_{22}}{\partial u \partial v}=0 .
$$

It is possible by a change of asymptotic parameters of $S$ to reduce (29) to

$$
\pi_{11}=\pi_{22} \text {. }
$$

Combining the last relation with (28) gives

$$
\beta_{u}=\gamma_{v} .
$$

That is, the surface $S$ is a Jonas surface $\left({ }^{6}\right)$. Moreover from (5) and (30) it follows that the developables of $L$ cut $S$ in the Jonas net:

$$
d u^{2}-d v^{2}=0
$$

On account of (25) we find immediately

$$
\beta_{u}^{\prime}=\gamma_{v}^{\prime}
$$

Hence the surface $S^{\prime}$ is also a Jonas surface.

Secondly, we show that the developables of $L$ cut $S^{\prime}$ also in the Jonas net.

Differentiating $x_{u}^{\prime}$ given in (23) with respect to $v$, we obtain

$$
e^{\theta} x_{u v}^{\prime}=\pi_{11} \gamma x_{u}+\pi_{22} \beta x_{v}+p x,
$$

the exact value of $p$ not being necessary at present.

In virtue of this relation and (23) the congruence $L$ is determined by the points $x^{\prime}$ and $\bar{x}$, defined as follows:

$$
\bar{x}=x^{\prime}+R\left(x_{u v}^{\prime}-l_{1}^{\prime} x_{v}^{\prime}-l_{2}^{\prime} x_{u}^{\prime}\right),
$$

wherein

$$
l_{1}^{\prime}=\frac{\pi_{11}}{\pi_{22}} \gamma, \quad l_{2}^{\prime}=\frac{\pi_{22}}{\pi_{11}} \beta .
$$

Since $\pi_{11}=\pi_{22}, \beta_{u}=\gamma_{v}$ we have

(6) Cf. Fubini and Čech, loc. cit. p. 106. 


$$
l_{1 v}^{\prime}=l_{2 u}^{\prime}
$$

Hence $L$ is also conjugate to $S^{\prime}$.

In view of (25), the fundamental quantities of $S^{\prime}$ are now found to be

$$
\begin{array}{lll}
\beta^{\prime}=\beta-\partial \log \pi_{11} / \partial v, & \theta_{u}^{\prime}=-\theta_{u}+\partial \log \pi_{11} / \partial u, & p_{11}^{\prime}=\pi_{11} \\
\gamma^{\prime}=\gamma-\partial \log \pi_{11} / \partial u, & \theta_{v}^{\prime}=-\theta_{v}+\partial \log \pi_{11} / \partial v, & p_{22}^{\prime}=\pi_{11}
\end{array}
$$

and

$$
\begin{aligned}
\pi_{11}^{\prime}=\pi_{11}+\beta_{v} & -\frac{\partial^{2} \log \pi_{11}}{\partial v^{2}}+\frac{\partial \log \pi_{11}}{\partial v}\left(\beta-\frac{\partial \log \pi_{11}}{\partial v}\right) \\
& -\theta_{v}\left(\beta-\frac{\partial \log \pi_{11}}{\partial v}\right), \\
\pi_{22}^{\prime}=\pi_{22}+\gamma_{u} & -\frac{\partial^{2} \log \pi_{11}}{\partial u^{2}}+\frac{\partial \log \pi_{11}}{\partial u}\left(\gamma-\frac{\partial \log \pi_{11}}{\partial u}\right) \\
& -\theta_{u}\left(\gamma-\frac{\partial \log \pi_{11}}{\partial u}\right) .
\end{aligned}
$$

By (5), the developables of $L$ cut $S^{\prime}$ in the following net:

$$
\begin{aligned}
\left(\pi_{11}^{\prime}-l_{1 u}^{\prime}+l_{1}^{\prime} \theta_{u}^{\prime}-l_{2}^{\prime} \beta^{\prime}-\right. & \left.l_{1}^{\prime 2}\right) d u^{2} \\
& +\left(-\pi_{22}^{\prime}+l_{1 v}^{\prime}-l_{2}^{\prime} \theta_{v}^{\prime}+l_{1}^{\prime} \gamma^{\prime}+l_{2}^{\prime 2}\right) d v^{2}=0 .
\end{aligned}
$$

In virtue of (35), (37), (38) and the second equation of (2), we find that the net defined by the last equation is precisely the net:

$$
d u^{2}-d v^{2}=0 .
$$

This completes the proof of the first part of Theorem II.

We now concern ourselves with the converse problem in Theorem II, that is, we wish to determine, for a given Jonas surface $S$, all congruences with the properties there quoted.

By the preceding discussions we conclude that if, for a given Jonas surface $S$, we could, by changing the factor of proportionality of $x$, cause the coefficients of (1) to be related by the formulas

$$
\pi_{11}=\pi_{22}, \quad \beta_{u}=\gamma_{v}, \quad \pi_{11 u v}=\left(\beta_{u}+\theta_{u v}\right) \pi+\beta \pi_{11 u}+\gamma \pi_{11 v}
$$

then the congruence of the lines joining $x$ and $(\rho x)_{u v}$ is the required congruence. Let $\bar{\beta}, \bar{\gamma}, \bar{\theta}_{u}, \bar{\theta}_{v}, \bar{p}_{11}, \bar{p}_{22}$ be the new fundamental quantities of $S$, then these quantities and the original fundamental quantities of $S$ must satisfy the relations similar to those given in (19). Hence the required function $\rho$ must be a solution of the following system of differential equations: 


$$
\bar{\pi}_{11 u v}-\bar{\pi}_{11} \bar{\theta}_{u v}-\beta \bar{\pi}_{11 u}-\gamma \bar{\pi}_{11 v}-\bar{\pi}_{11} \beta_{u}=0, \quad \bar{\pi}_{11}=\bar{\pi}_{22},
$$

which is completely integrable in virtue of $\beta_{u}=\gamma_{v}$. We write the second equation of (41) in the form

$$
\rho_{u u}+\beta \rho_{v}-\theta_{u} \rho_{u}-\rho \pi_{11}=k, \quad \rho_{v v}+\gamma \rho_{u}-\theta_{v} \rho_{v}-\rho \pi_{22}=k,
$$

where $k$ denotes an arbitrary function of $u$ and $v$. Combining (42) and (411) we see that the initial values of $\rho, \rho_{u}, \rho_{v}, \rho_{u v}, k, k_{u}, k_{v}, k_{u u}$ of a solution of (41) may be assigned arbitrarily. Hence for any Jonas surface, there exist $\infty^{8}$ congruences having the properties stated in Theorem II.

The proof of Theorem II is now completed.

It is concluded that from any given Jonas surface, we can deduce $\infty^{8}$ new surfaces of the same type with asymptotic tangents intersecting in pairs. The fundamental differential equations of each are

$$
\begin{aligned}
& x_{u u}=\left(\beta-\frac{\partial \log \pi_{11}}{\partial v}\right) x_{v}+\left(-\theta_{u}+\frac{\partial \log \pi_{11}}{\partial u}\right) x_{u}+\pi_{11} x, \\
& x_{v v}=\left(\gamma-\frac{\partial \log \pi_{11}}{\partial u}\right) x_{u}+\left(-\theta_{v}+\frac{\partial \log \pi_{11}}{\partial v}\right) x_{v}+\pi_{11} x,
\end{aligned}
$$

which are completely integrable because of (40) as well as the conditions of integrability (2) of the original surface.

Hence if the asymptotic curves of both $S, S^{\prime}$ and the focal surfaces of $L$ correspond, then both the surfaces $S$ and $S^{\prime}$ are characterized by the following conditions:

$$
\beta_{v}=\gamma_{u}, \quad \beta_{u}=\gamma_{v} .
$$

A special case of Theorem II is the following:

If a surface $S$ is generated by the harmonic conjugate point of the point of intersection of a congruence $L$ with a fixed plane with respect to the corresponding foci of $L$ and is conjugate to $L$, then $S$ is a Jonas surface and the developables of $L$ cut $S$ in the Jonas net.

6. We are now in a position to discuss the special class of congruences which we have excluded in the preceding paragraphs, that is, when the developables of $L$ are indeterminate. In this case, $l_{1 v}=l_{2 u}$. By changing the factor of proportionality the congruence is defined by $x$ and $\bar{x}, \bar{x}$ being defined by

$$
\bar{x}=x+R x_{u v},
$$

with

$$
\pi_{11}=\pi_{22}=0 .
$$

It is known that, in this case, all lines of the congruence $L$ pass through a 
fixed point. For this congruence we can deduce a result similar to Lemma 1, namely,

If $S$ and $S^{\prime}$ are distinct transversal surfaces of a congruence $L$ and the asymptotic u-tangents of $S$ intersect the corresponding $u$-tangents of $S^{\prime}$, then all lines of $L$ pass through a fixed point. Conversely, if we have a congruence $L$ with its lines passing through a fixed point, the asymptotic u-tangents of $S$ intersect the corresponding $u$-tangents of $S^{\prime}$, where $S$ and $S^{\prime}$ are surfaces generated by any two distinct points $(x)$ and $\left(x^{\prime}\right)$ situated on each generator of the congruence (these two surfaces are said to be perspective).

For a pair of perspective surfaces we state also the following result which may be considered as a generalization of Koenig's theorem for plane asymptotic nets:

If two surfaces $S, S^{\prime}$ are perspective and if the asymptotic curves of $S$ correspond to a conjugate net $\Omega$ of $S^{\prime}$ under this perspectivity, then $\Omega$ is a net with equal invariants. Conversely, any net with equal invariants is perspective to the asymptotic net of another surface.

The lines joining each pair of corresponding points of $S$ and $S^{\prime}$ form a congruence $L$ with indeterminate developables. By changing the factor of proportionality we can define this congruence $L$ by

$$
x^{\prime}=e^{-\theta}\left(R x+x_{u v}\right), \quad \pi_{11}=\pi_{22}=0,
$$

where $\left(x^{\prime}\right)$ is a point of $S^{\prime}$ corresponding the point $(x)$ of $S$. By a simple calculation we have

$$
\begin{aligned}
x_{u}^{\prime} & =e^{-\theta}\left[\left(p_{u}-\theta_{u} p\right) x+p x_{u}\right], \\
x_{v}^{\prime} & =e^{-\theta}\left[\left(p_{v}-\theta_{v} p\right) x+p x_{v}\right], \\
x_{u v}^{\prime} & =e^{-\theta}\left[\left(p_{v}-\theta_{v} p\right) x_{u}+\left(p_{u}-\theta_{u} p\right) x_{v}+p x_{u v}+q x\right],
\end{aligned}
$$

where we have put

$$
p=R+\theta_{u v}+\beta \gamma,
$$

and $q$ is a function which has no importance for us.

By means of this set of equations, we obtain

$$
x_{u v}^{\prime}=\left(\frac{\partial \log p}{\partial v}-\theta_{v}\right) x_{u}^{\prime}+\left(\frac{\partial \log p}{\partial u}-\theta_{u}\right) x_{v}^{\prime}+l x^{\prime}+m x .
$$

Thus follows the first part of our theorem. The converse theorem can be proved in a similar way.

7. We have shown in Lemma 1 (\$3) that if $S$ is conjugate to $L$, the asymptotic $v$-tangents ( $u$-tangents) of $S$ intersect the corresponding $u$-tangents (v-tangents) of $S^{\prime}$. The lines joining each pair of corresponding points of intersection form a congruence $L^{\prime}$ defined by the points $x_{u}^{\prime}, x_{v}^{\prime}$ where 


$$
\begin{aligned}
& x_{u}^{\prime}=e^{-\theta}\left[\left(\pi_{22} \beta-\pi_{11 v}\right) x+\pi_{11} x_{v}\right], \\
& x_{v}^{\prime}=e^{-\theta}\left[\left(\pi_{11} \gamma-\pi_{22 u}\right) x+\pi_{22} x_{u}\right] .
\end{aligned}
$$

The reciprocal congruence $\bar{L}$ of $L^{\prime}$ with respect to $S$ is then defined by

$$
-l_{1}=\frac{\pi_{11}}{\pi_{22}} \gamma-\frac{\partial \log \pi_{22}}{\partial u}, \quad-l_{2}=\frac{\pi_{22}}{\pi_{11}} \beta-\frac{\partial \log \pi_{11}}{\partial v} .
$$

Hence we arrive at the following theorem:

The congruence $\bar{L}$ is also conjugate to $S$ when and only when the developables of $L$ cut $S$ in a net with equal point invariants.

In the second place we deduce the condition that the congruence $L$ (harmonic conjugate of $S$ with respect to $S$ ) be conjugate to $S^{\prime}$.

Let the asymptotic curves of $S^{\prime}$ be defined by the following equation:

$$
A d u^{2}+2 B d u d v+C d v^{2} \equiv F d \bar{u} d \bar{v}=0 .
$$

If $L$ is also conjugate to $S^{\prime}$, then, by Lemma 1 (\$3), the asymptotic $\bar{u}$ tangents of $S^{\prime}$ intersect the corresponding $\bar{v}$-tangents of $S$ and therefore $\left(x^{\prime}, x_{\bar{u}}^{\prime}, x, x_{\bar{v}}\right)=0$. By a simple calculation, we find the required necessary and sufficient condition as

$$
\pi_{11} \frac{\partial u}{\partial \bar{u}} \frac{\partial u}{\partial \bar{v}}=\pi_{22} \frac{\partial v}{\partial \bar{u}} \frac{\partial v}{\partial \bar{v}}
$$

or

$$
A / \pi_{11}=C / \pi_{22}
$$

8. This paper concludes with the conditions that a congruence $L$ conjugate to a surface $S$ should be $W$. The congruence $L$, being conjugate to $S$, is defined by the Plücker coordinates of its lines, namely $r=\left(x, x_{u v}\right)$. By differentiation we have

$$
\begin{aligned}
r_{u u}= & 3 \theta_{u}\left(x_{u}, x_{u v}\right)+\beta\left(x_{v}, x_{u v}\right)+2 \pi_{11}\left(x_{u}, x_{v}\right) \\
& +\left[\pi_{11 v}-\pi_{22} \beta+3 \theta_{u}\left(\theta_{u v}+\beta \gamma\right)\right]\left(x, x_{u}\right) \\
& +\left[\pi_{11 u}+\beta\left(\theta_{u v}+\beta \gamma\right)+\theta_{u} \pi_{11}\right]\left(x, x_{v}\right) \\
& +\left(\pi_{11}+p_{11}+\theta_{u}^{2}+\theta_{u u}\right)\left(x, x_{u v}\right), \\
r_{v v}= & \gamma\left(x_{u}, x_{u v}\right)+3 \theta_{v}\left(x_{v}, x_{u v}\right)-2 \pi_{22}\left(x_{u}, x_{v}\right) \\
& +\left[\pi_{22 v}+\gamma\left(\theta_{u v}+\beta \gamma\right)+\theta_{v} \pi_{22}\right]\left(x, x_{u}\right) \\
& +\left[\pi_{22 u}-\pi_{11} \gamma+3 \theta_{v}\left(\theta_{u v}+\beta \gamma\right)\right]\left(x, x_{v}\right) \\
& +\left(\pi_{22}+p_{22}+\theta_{v}^{2}+\theta_{v v}\right)\left(x, x_{u v}\right),
\end{aligned}
$$


and the values of $r_{u}, r_{v}, r_{u v}$ are given by (13).

If $L$ is $W$ the quantities $r, r_{u}, r_{v}, r_{u v}, r_{u u}, r_{v v}$ satisfy a linear relation, that is, the determinant formed by the coefficients of $\left(x_{u}, x_{u v}\right),\left(x_{v}, x_{u v}\right),\left(x_{u}, x_{v}\right)$, $\left(x, x_{u}\right),\left(x, x_{v}\right)$ of $r_{u}, r_{v}, r_{u v}, r_{u u}, r_{v v}$ is to be equal to zero. By a simple calculation, we find the required condition as follows:

$$
\pi_{22 u}\left[\frac{\partial \log \pi_{11} \pi_{22}}{\partial u}-2\left(\theta_{u}+\frac{\pi_{11}}{\pi_{22}} \gamma\right)\right]
$$

$$
=\pi_{11 v}\left[\frac{\partial \log \pi_{11} \pi_{22}}{\partial v}-2\left(\theta_{v}+\frac{\pi_{22}}{\pi_{11}} \beta\right)\right] .
$$

In particular, surfaces for which the projective normal congruence is $W$ are characterized by equation (46), where the function $\theta$ takes the value

$$
\theta=\log \beta \gamma \text {. }
$$

National University of Cheriang, Meitan, Kweichow, China. 